ARDB 170

1998

\title{
Chapter 3. Article for Handbook of Accelerator Physics and Technology
}

\author{
J.E. Spencer
}

Stanford Linear Accelerator Center, Stanford University, Stanford, CA 94309

Work supported by Department of Energy contract DE-AC03-76SF00515. 


\section{Chapter 3. Article for Handbook of Accelerator Physics and Technology}

\subsubsection{Bhabha Scattering $\left(\mathrm{e}^{+} \mathrm{e}^{-} \rightarrow \mathrm{e}^{+} \mathrm{e}^{-}\right)$ J.E. Spencer, SLAC}

The elastic scattering of electrons and positrons proceeds via one boson exchange and annihilation diagrams (the $\mathrm{u}$ and $\mathrm{t}$ channels for $\mathrm{e}^{-} \mathrm{e}^{-} \rightarrow \mathrm{e}^{-} \mathrm{e}^{-}$):
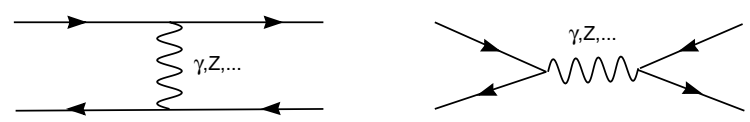

The differential cross section with one photon exchange for unpolarized beams in the $\mathrm{CM}$ is [1]

$$
\begin{gathered}
\frac{d \sigma_{\text {Born }}^{\text {QED }}}{d \Omega}\left(\mathrm{e}^{+} \mathrm{e}^{-} \rightarrow \mathrm{e}^{+} \mathrm{e}^{-}\right) \\
=\frac{\alpha^{2}}{2 s}\left[\frac{1+\cos ^{4} \frac{\theta}{2}}{\sin ^{4} \frac{\theta}{2}}-\frac{2 \cos ^{4} \frac{\theta}{2}}{\sin ^{2} \frac{\theta}{2}}+\frac{1}{2}\left(1+\cos ^{2} \theta\right)\right]
\end{gathered}
$$

when $\sqrt{s} \equiv\left(p_{1}+p_{2}\right) c \gg m c^{2}$ (typically $s=4 E_{b}^{2}$ ). Units natural to relativistic quantum theory are used ( $c=\hbar=1)$ when no confusion is likely.

The large forward cross section is useful for monitoring $\mathrm{e}^{ \pm}$luminosity while the larger angle scattering is used for electroweak measurements e.g. using the $Z$ annihilation diagram. Based on lepton universality, the $\mathrm{e}^{ \pm}$annihilation diagram corresponds to $\mathrm{e}^{+} \mathrm{e}^{-} \rightarrow \mu^{+} \mu^{-}$and more generally, for elementary fermions $f$ of charge $\mathrm{e} Q_{f}$,

$$
\frac{d \sigma}{d \Omega}\left(l^{+} l^{-} \rightarrow f^{+} f^{-}\right)=\frac{\alpha^{2}}{4 s}\left(1+\cos ^{2} \theta\right) Q_{f}^{2}
$$

Integrating gives the (partial) total cross section of the expected luminosity or background rates in annular detectors. For example, for the u-quark with $Q_{f}=2 / 3$ and muons,

$$
\begin{aligned}
\sigma\left(\mathrm{e}^{+} \mathrm{e}^{-} \rightarrow \mathrm{u}^{+} \mathrm{u}^{-}\right) & =\frac{4}{9} \frac{\pi \alpha^{2}}{2 s}\left[\cos \theta+\frac{1}{3} \cos ^{3} \theta\right]_{\theta_{2}}^{\theta_{1}} \\
\sigma\left(\mathrm{e}^{+} \mathrm{e}^{-} \rightarrow \mu^{+} \mu^{-}\right) & =\frac{9}{4} \sigma\left(\mathrm{e}^{+} \mathrm{e}^{-} \rightarrow \mathrm{u}^{+} \mathrm{u}^{-}\right)
\end{aligned}
$$

where $4 \pi \alpha^{2}=261 \mathrm{GeV}^{2} \mathrm{nb}$.
Møller scattering The corresponding cross section for $\mathrm{e}^{ \pm} \mathrm{e}^{ \pm} \rightarrow \mathrm{e}^{ \pm} \mathrm{e}^{ \pm}$is [2]

$$
\begin{gathered}
\frac{d \sigma_{\text {Born }}^{\text {QED }}}{d \Omega}\left(\mathrm{e}^{ \pm} \mathrm{e}^{ \pm} \rightarrow \mathrm{e}^{ \pm} \mathrm{e}^{ \pm}\right) \\
=\frac{\alpha^{2}}{2 s}\left(\frac{1+\cos ^{4} \frac{\theta}{2}}{\sin ^{4} \frac{\theta}{2}}+\frac{1+\sin ^{4} \frac{\theta}{2}}{\cos ^{4} \frac{\theta}{2}}+\frac{2}{\sin ^{2} \frac{\theta}{2} \cos ^{2} \frac{\theta}{2}}\right)
\end{gathered}
$$

Luminosity monitoring with Bhabha (Møller) scattering BS is used to monitor or calibrate luminosity by selecting events with two oppositely charged tracks with high momentum (small $p_{t}$ elastic) and small acollinearity angle that originate at the IP. Various contaminations such as muon pairs are statistically subtracted.

A quite good approximation for the singles rate (or pair coincidence rate) for an annular detector subtending an angular range $\delta \theta\left(\theta_{1}\right.$ to $\left.\theta_{2}\right)$ near the IP - ignoring field effects from the detector or beam optics is

$$
\sigma(\delta \theta) \approx \frac{4 \pi \alpha^{2}}{s}\left[\frac{1}{\sin ^{2}\left(\frac{\theta}{2}\right)}+4 \ln \left(\sin \frac{\theta}{2}\right)\right]_{\theta_{2}}^{\theta_{1}}
$$

For small angles,

$$
\sigma(\delta \theta) \approx 4.2\left(\frac{250 \mathrm{GeV}}{E_{b}}\right)^{2} \frac{\theta_{2}^{2}-\theta_{1}^{2}}{\theta_{1}^{2} \theta_{2}^{2}} \mathrm{pb}
$$

If the predicted rate is reasonable, we can then consider the backgrounds predicted in the beambeam simulations to estimate the signal-to-noise ratio. Increasing $\theta_{2}$ is usually not effective. The expected Bhabha rate is then given by $\left.\mathcal{L} \sigma(\delta \theta)\right|_{\theta_{1}} ^{\theta_{2}}$.

It is fair to ignore the solenoid field if there is no crossing angle between the colliding beams or strong disruption from the beam-beam effects. However, if backgrounds are important, it is useful to go outside of the solenoid to permit some degree of beam analysis. To account for such conditions including finite beam size and divergence effects there are Monte Carlo codes (see 
e.g. ABLE, BHLUMI and others) that can be used to simulate the beams and their interactions at the IP for use with tracking codes such as MAD or TRANSPORT for optics design and electromagnetic shower codes such as GEANT or EGS4 for detector design and background simulation.

A semi-analytical way to use such codes is to transport the generated events (for the assumed conditions and perturbed by the beam-beam interaction) outside the primary detector. The resulting rate of scattered pair events and singles is then a function of the effective, disrupted luminosity, the acceptance of the detector and the intervening beam optics. A standard approximation, for the rate in a rectangular detector outside a solenoid, is

$$
\begin{aligned}
R_{\mathrm{BS}}^{\mathrm{QED}} & =\mathcal{L} \frac{16 \alpha^{2}}{s} \iint \frac{d \Omega}{\theta^{4}} \\
& =\mathcal{L} \frac{16 \alpha^{2}}{s} \int_{x^{\prime}} \int_{y^{\prime}} \frac{d x^{\prime} d y^{\prime}}{\left(x^{\prime 2}+y^{\prime 2}\right)^{2}}
\end{aligned}
$$

where $\theta=\sqrt{{x^{\prime}}^{2}+y^{\prime 2}}, \tan \phi=y^{\prime} / x^{\prime}$, and $x^{\prime}, y^{\prime}$ are the angles in cartesian coordinates.

For a transverse point source and dispersion free transport from the IP to the detector, the position of a particle at the detector is $x_{d}=R_{12} x^{\prime}$ and $y_{d}=R_{34} y^{\prime}$ where $R_{12}$ and $R_{34}$ can be calculated with a tracking code. Substituting the BS detector's coordinates in the optical reference system gives the corresponding integration limits to compute the expected rates,

$$
\begin{aligned}
R_{\mathrm{BS}}^{\mathrm{QED}} & =\mathcal{L} \frac{4 \alpha^{2}}{s}\left[-\frac{1}{x^{\prime} y^{\prime}}\right. \\
& \left.-\left(\frac{1}{x^{\prime 2}}+\frac{1}{y^{\prime 2}}\right) \tan ^{-1}\left(\frac{y^{\prime}}{x^{\prime}}\right)\right]\left._{y_{1}^{\prime}}^{y_{2}^{\prime}}\right|_{x_{1}^{\prime}} ^{x_{2}^{\prime}}
\end{aligned}
$$

A detector whose area is $\mathrm{L}_{x} \mathrm{~L}_{y}$ might cover the area corresponding to $\mathrm{x}_{1}^{\prime}=\mathrm{X} / R_{12}$ to $\mathrm{x}_{2}^{\prime}=\left(\mathrm{X}+\mathrm{L}_{x}\right) / R_{12}$ and $\mathrm{y}_{1}^{\prime}=-\mathrm{L}_{y} / 2 R_{34}$ to $\mathrm{y}_{2}^{\prime}=\mathrm{L}_{y} / 2 R_{34}$. The values of $\mathrm{X}$ (and/or $\mathrm{Y}$ ) and $\mathrm{L}_{x, y}$ are chosen to avoid the incoming and outgoing disrupted beams and backgrounds.

Radiative Bhabha scattering $\left(\mathrm{e}^{+} \mathrm{e}^{-} \rightarrow \mathrm{e}^{+} \mathrm{e}^{-} \gamma\right)$
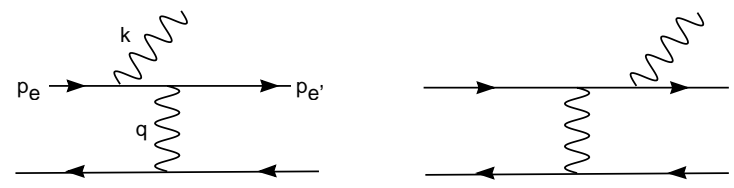

A free, charged particle is expected to radiate when scattered or accelerated: 'it never happens that a scattering event is unaccompanied by the emission of quanta' [3]. Pure Bhabha scattering occurs at extremely small and presumably unresolvable angles for leptons. For the RBS photons, $\theta_{k} \sim \mathcal{O}(1 / \gamma)$. For the radiating lepton,

$$
\theta_{l^{\prime}} \sim \frac{1}{\gamma} \frac{1-w}{w} \sim \frac{1}{\gamma} \frac{E_{k}}{E_{b}-E_{k}}
$$

where $w \approx E_{e^{\prime}} / E_{b}$. The RBS differential cross section in lowest order in the ultrarelativistic limit for the outgoing lepton is [4]

$$
\begin{gathered}
\frac{d \sigma_{\mathrm{e}^{-}}}{d w}\left(\mathrm{e}^{ \pm} \mathrm{e}^{\mp} \rightarrow \mathrm{e}^{ \pm} \mathrm{e}^{\mp} \gamma\right) \\
=\frac{4 \alpha r_{e}^{2}}{1-w}\left(1+w^{2}-\frac{2}{3} w\right)\left(\ln \frac{s}{m_{e}^{2}} \frac{w}{1-w}-\frac{1}{2}\right)
\end{gathered}
$$

and for the photons [4], with $y=E_{k} / E_{b}$,

$$
\begin{gathered}
\frac{d \sigma_{\gamma}}{d y}\left(\mathrm{e}^{ \pm} \mathrm{e}^{\mp} \rightarrow \mathrm{e}^{ \pm} \mathrm{e}^{\mp} \gamma\right) \\
=\frac{4 \alpha r_{e}^{2}}{y}\left[y^{2}+\frac{4}{3}(1-y)\right]\left(\ln \frac{s}{m_{e}^{2}} \frac{1-y}{y}-\frac{1}{2}\right)
\end{gathered}
$$

where $4 \alpha r_{e}^{2}=2.32 \mathrm{mb}$. Using a finite bunch size correction [5], a good approximation expected for the radiated photon power for some bandwidth $\delta y$ into a forward detector is

$$
\begin{aligned}
& P_{\gamma} \approx \mathcal{L} E_{b}\left(\frac{4}{3} \alpha r_{e}^{2}\right) {\left[2+\ln \frac{\sigma_{x} \sigma_{y}}{\lambda_{c}\left(\sigma_{x}+\sigma_{y}\right)}\right] } \\
& \times\left[y^{3}-2 y^{2}+4 y\right]_{y_{\min }}^{y_{\max }}
\end{aligned}
$$

where $\lambda_{c}=r_{e} / \alpha$ and $\sigma$ is the rms beam size. The value $y_{\min } \rightarrow 0$ is never required in the above expressions because of cuts or detector resolution. Some conclusions are:

- Elastic BS decreases inversely with $s=4 E_{b}^{2}$. - RBS effects typically increase logarithmically with $s / m^{2}$.

- Photons are emitted within an angle $\theta_{\gamma}=$ $m / E_{b}$ with the particles.

- Their spectrum will run up to $\sim E_{b}$ and vary as $1 / E_{b}$.

- Hard scattering corresponds to angles outside the $m / E_{b}$ cone.

- Typical beam divergence angles and luminosity monitors are $\gg \theta_{\gamma}$.

- Virtually all scattered particles that are detected are hard.

- Initial state radiation can't be explicitly distinguished. 
- This is true for the final state when the radiative event is untagged.

- This implies the outgoing scattered particles are not generally collinear.

- It also implies that the $\mathrm{cm}$ and lab systems are no longer the same.

- Beam-beam disruption will broaden the acollinearity distribution.

- Most experiments based on BS need to include radiative effects.

Electrodynamics suggests other radiative effects that need to be considered for BS in an external field or non-free environment. A more complete discussion is available [6] including comparisons to data, ranges of applicability of expressions and the importance of such radiative effects.

\section{References}

[1] H.J. Bhabha, Proc. Roy. Soc. 154 (1935) 195

[2] C. Møller, Ann. d. Physik 14 (1932) 568

[3] J. Schwinger, PR 75 (1949) 898

[4] G. Altarelli, F. Bucella, Nuovo Cim. 34 (1964) 1337; G. Altarelli, B. Stella, Lett. Nuovo Cim. 9 (1974) 416

[5] A.E. Blinov et al, Phys. Lett. 113B (1982) 423

[6] J.E. Spencer, SLAC-ARDB Rept. (1997) 